\title{
How choice ecology influences search in decisions from experience
}

\author{
Tomás Lejarraga ${ }^{\mathrm{a}, \mathrm{d}, *}$, Ralph Hertwig ${ }^{\mathrm{b}, \mathrm{c}}$, Cleotilde Gonzalez ${ }^{\mathrm{d}}$ \\ a Departament d'Economia de l'Empresa, Universitat de les Illes Balears, Ctra. Valldemossa Km. 7.5, 07122 Palma de Mallorca, Illes Balears, Spain \\ ${ }^{\mathrm{b}}$ Department of Psychology, University of Basel, Missionsstrasse 64A, 4055 Basel, Switzerland \\ ${ }^{\mathrm{c}}$ Max Planck Institute for Human Development, Lentzeallee 94, 14195 Berlin, Germany \\ ${ }^{\mathrm{d}}$ Dynamic Decision Making Laboratory, Department of Social and Decision Sciences, Carnegie Mellon University, 5000 Forbes Avenue, Pittsburgh, PA 15213, \\ United States
}

\section{A R T I C L E I N F O}

\section{Article history:}

Received 19 December 2011

Revised 2 June 2012

Accepted 6 June 2012

Available online 2 July 2012

\section{Keywords:}

Decisions from experience

Search

Sampling

Loss

Risk

\begin{abstract}
A B S T R A C T
Research into human decision-making has often sidestepped the question of search despite its importance across a wide range of domains such as search for food, mates, allies, visual targets or information. Recently, research on decisions from experience has made progress in finding out how individual characteristics shape search for information. Surprisingly little is known, however, about how the properties of the choice ecology shape people's search. To fill this void, we analyzed how two key ecological properties influence search effort: domain of choice (gains vs. losses) and experienced variance (variance vs. no variance). Many people search longer when facing the prospect of losses relative to gains. Moreover, most people search more in options in which they experience variance relative to options they experience as invariant. We conclude that two factors that have been identified as important determinants of choice also influence search of information.
\end{abstract}

(c) 2012 Elsevier B.V. All rights reserved.

\section{Introduction}

For Herbert Simon, one of the most influential economists and cognitive scientists of the second half of the 20th century, search was perhaps the key cognitive process of human life. For instance, in his work with Allen Newell, he conceptualized problem-solving as search for a path through a problem space with the goal of finding a route linking the problem's initial state to the goal state via intermediate steps and subgoals (Newell \& Simon, 1972). Search also figures prominently in his notion of "satisficing" (Simon, 1956, 1990). Satisficing is a method one can recruit when choosing from a set of alternatives (e.g., job candidates, mates) that one encounters sequentially and when little is known about the possible alternatives further down the road. Satisficing means that the decision

\footnotetext{
* Corresponding author at: Departament d'Economia de l'Empresa, Universitat de les Illes Balears, 07122 Palma de Mallorca, Illes Balears, Spain. Tel.: +34 971 172438; fax: +34971 132390 .

E-mail address: tomas.lejarraga@uib.eu (T. Lejarraga).
}

maker sets an aspiration level and terminates sequential search for alternatives as soon as one is found whose value exceeds the aspiration level. Finally, in a grand metaphor of human life, Simon (1996) depicted our existence as search through a "life's maze" (p. 166), representing a multiplicity of needs and wants, and the need to "survive without drawing upon superhuman powers of intelligence and computation" (p. 175).

Notwithstanding the importance of search as a behavioral and cognitive process across a wide range of domains-foraging for food, visual search for targets, memory search in recall and recognition tasks, the search for information on the web, and the search for mates in social environments (Todd, Hills, \& Robbins, 2012)-research on behavioral decision-making (e.g., research on inductive and logical reasoning) and cognition (e.g., research on moral reasoning) has often sidestepped questions of search by using tasks in which summary information is conveniently displayed before the respondents. Past research on risky choice exemplifies this methodological proclivity. Decision researchers, using choice between monetary 
gambles as a canonical model for risky choice, have grown accustomed to presenting their respondents with a complete description of the problem, spelling out all outcomes and their probabilities (see Weber, Shafir, \& Blais, 2004). ${ }^{1}$ Respondents are thus asked to make decisions from description (Hertwig, Barron, Weber, \& Erev, 2004). Consequently, cognitive search has being reduced to a whiff of searchsequentially proceeding through the described information, with internal search (in memory) and external search (in environments) made largely unnecessary. To investigate such decisions from description, researchers have devised ingenious methods (e.g., Willemsen \& Johnson, 2010) to make observable what little is left of cognitive search. With the recent advent of investigations of what has been termed the description-experience gap in risky choice (see Hertwig \& Erev, 2009), however, search has been moved to the center of scientific interest.

\section{The description-experience gap}

Everyday life rarely affords us the opportunity to peruse descriptions of probability distributions over outcomes. One of the few exceptions is the daily media weather forecast stating chance of precipitation (Gigerenzer, Hertwig, van den Broek, Fasolo, \& Katsikopoulos, 2005). In contrast, when people decide whether to take out a loan, contemplate their choices during speed-dates (Todd, Penke, Fasolo, \& Lenton, 2007), or in a split-second decide whether to cross the street in front of oncoming vehicles (Hoffrage, Weber, Hertwig, \& Chase, 2003), there are no tabulated risks to consult. Instead, people either rely on past experiences with these options, or engage in the options' on-line exploration, thus ultimately making decisions from experience rather than decisions from description (Hertwig et al., 2004). Both kinds of decisions can be understood as opposite entries on a continuum of uncertainty about what one is choosing between. In Knight (1921) time-honored terminology, decisions from description involve a priori probabilities (based, for instance, on transparent random mechanisms such as a fair die), whereas decisions from experience involve statistical probabilities; the latter must be gauged, if possible, from experience.

Decisions from experience have been studied based on several experimental paradigms (Hertwig \& Erev, 2009). We will focus on the sampling paradigm. Respondents face two buttons on a computer screen, each representing an initially unknown payoff distribution (or option; henceforth, we use the terms "option" and "payoff distribution" synonymously). For instance, one button represents an option $A$ involving the outcomes 4 and 0 , with 4 occurring with a probability of .2 , whereas the other button represents an option $B$ offering 3 and 0 , with 3 occurring with a probability of .25. Respondents can overcome their ignorance by exploring the payoff distributions. Specifically,

\footnotetext{
1 Interestingly, in the 1950s and 1960s, the experimental marketplace was not yet monopolized by investigations of choices between described monetary gambles. Decision scientists then examined, for example, how people learn the probability distribution over outcomes through repeated trial-by-trial feedback (e.g., Edwards', 1961, “probability learning in 1000 trials" article).
}

clicking a button results in a random draw from the respective distribution. Respondents sample as many outcomes as they wish and only then decide from which distribution to make a single draw for real (i.e., an incentivized draw). This final decision can then be compared to those reached when the same options are described explicitly. Across different experimental paradigms, a robust and systematic gap between experience- and description-based choices has emerged (see Hertwig \& Erev, 2009), for a review). It can be summarized as follows: In decisions from experience, people behave as if the rare events have less impact than they deserve according to their objective probabilities, whereas in decisions from description people behave as if the rare events have more impact than they deserve (Kahneman \& Tversky, 1979; also see Ludvig \& Spetch, 2011), for a demonstration of the description-experience gap beyond rare events).

\section{Search in decisions from experience}

Researching decisions from experience offers a great advantage relative to investigating description-based risky choices: The experimental paradigms lay wide open what otherwise is difficult to observe: people's search for information. Consequently, we have recently learned much about the psychology of search in decisions from experience. We now know, for instance, that in the sampling paradigm (a) people tend to rely on fairly small samplesranging mostly from 11 to 19 draws, amounting to nearly $7 \pm 2$ draws from each distribution-thereby attenuating the impact of rare events (see Hau, Pleskac, \& Hertwig, 2010, Table 1); (b) people respond to incentives such that increasing the monetary stakes substantially boosts sampling efforts (Hau, Pleskac, Kiefer, \& Hertwig, 2008); (c) small samples amplify the difference between the expected average earnings, thus making the options more distinct and choice easier (Hertwig \& Pleskac, 2008, 2010); (d) people's short-term memory capacity is positively correlated with the size of the drawn sample $(r=.36$; Rakow, Demes, \& Newell, 2008); (e) people endowed with high numeracy draw larger samples than those with low numeracy (Lejarraga, 2010); (f) people who report to have a high ability in rational thinking draw larger samples than those with low ability (Lejarraga, 2010); and (g) people tend to adopt one of two idealized search policies (piece-wise vs. round-wise search strategy, with the former involving more switching between options), with switching frequency being negatively correlated with sample size $(r=-.44)$ and positively associated with a decision strategy conducive to rare events being underweighted (Hills \& Hertwig, 2010).

In order to map the psychology of search in decisions from experience, one can examine the (a) cognitive properties of the decision maker (e.g., short-term memory capacity, rational abilities, search policies); (b) the properties of the choice ecology (e.g., loss vs. gain domain), and (c) the interaction of ecology and cognition (e.g., amplification effect; Hertwig \& Pleskac, 2010). As the brief review of past results illustrates, progress has been made in describing how cognitive properties shape search. Surprisingly little 
is known, however, about how ecological properties influence search. The goal of this paper is thus to find out how environmental properties-via an interplay with psychological constructs (e.g., loss aversion)-have an effect on search.

\section{Is sample size a function of the choice ecology?}

We focus on two properties of the choice ecology. Specifically, we investigate the extent to which search-in particular, search effort (i.e., sample size)-hinges on two key ecological properties, namely, domain and variance. Domain refers to the potential value of a payoff distribution's outcomes, specifically, whether the experienced outcomes represent gains or losses. Variance refers to whether the experienced outcomes from a payoff distribution reveal the presence of more than a single outcome or suggest lack of variability (and, by extension, risk). Investigating the impact of these properties on search allows us to also peek into the mind of the decision maker. If people adapt their search in response to initial information about domain and variance, we can also draw inferences about psychological constructs correlated with them, namely, loss and risk aversion. Next, we turn to these constructs.

\subsection{Do losses invoke more search than gains?}

One influential notion in psychological and economic research on decision making is loss aversion (Tversky \& Kahneman, 1992) according to which "people are more sensitive to the possibility of losing objects or money than they are to the possibility of gaining the same objects or amount of money" (Tom, Fox, Trepel, \& Poldrack, 2007, p. 515). It has been called upon to explain a diverse range of phenomena including the endowment effect (Kahneman, Knetsch, \& Thaler, 1990; Thaler, 1980), the status quo bias (Samuelson \& Zeckhauser, 1988), the equity premium puzzle (Benartzi \& Thaler, 1995), and the labor supply of cabdrivers (Camerer, Babcock, Loewenstein, \& Thaler, 1997). In the context of choice between monetary gambles, loss aversion suggests that because of people's sensitivity to losses relative to gains, most would not accept gambles with mixed symmetric outcomes (i.e., gaining or losing an amount $x$ with the same likelihood). Empirical evidence, however, appears not to support this prediction (for a review see Hochman \& Yechiam, 2011). Relatedly, in decisions from experience there is an ongoing debate about how to explain choices previously interpreted as indicative of loss aversion (e.g., Erev, Ert, \& Yechiam, 2008).

Notwithstanding the disputed empirical support for loss aversion on the level of choice, there is evidence that "losses lead to heightened autonomic responses, compared to equivalent gains (as indicated by pupil dilation and increased heart rate) even in situations where the average decision maker exhibits no loss aversion" (Hochman \& Yechiam, 2011, p. 140, and the review herein). People's response to negative events may thus unfold differently on different levels. On a physiological level, losses appear to trigger increased autonomic arousal relative to equivalent gains (Gonzalez, Dana, Koshino, \& Just, 2005), consistent with the interpretation that losses signal the presence of a threat. Such arousal, however, need not inevitably translate into behavioral choices indicative of elevated sensitivity to losses relative to gains.

There are intermediate levels between the initial physiological arousal and behavioral choice. One of them is search. Search in the sampling paradigm does not bind a person to choose a particular alternative; yet, exploring one alternative more than another may reflect attraction or vigilance toward it. Therefore, we investigate whether and to what extent the prospect of suffering a loss triggers more search than that of reaping a gain. If search is aligned with the physiological level, then the prospect of a loss will likely trigger more search than that of a gain. One likely reason is that searching more would permit individuals to gauge the payoff distribution and the risk of a loss more accurately. If search, however, is aligned with the final choice-and behavioral choice is less responsive to the threat of loss than our physiology-then the risk of a loss will not necessarily invoke more search than the chance of a gain.

\subsection{Does the experience of variability result in more search?}

A diverse set of theories of decision making under risk and uncertainty-ranging from expected utility theory (von Neumann \& Morgenstern, 1947), and risk return models (Markowitz, 1952) to prospect theory (Kahneman \& Tversky, 1979)-share the assumption that the likelihood with which a risky option is chosen is a function of the variability of the option's possible outcomes. In fact, a typical measure of risk is the second moment of the distribution of outcomes, that is, the variance of outcomes around the option's expected value. The higher the outcome variance of risky options (with otherwise equal expected values), that is, the riskier it is, the less likely people are to choose the options, and the lower their willingness to pay for them (Sharpe, 1964). This "payoff-variability effect" is also observed when people experience variance while choosing repeatedly from a set of options (Busemeyer \& Townsend, 1993; Erev \& Barron, 2005; Myers \& Sadler, 1960).

To the extent that outcome variance is also a psychologically suitable proxy for perceived risk in decisions from experience, it is likely to affect how people make them. Furthermore, experienced variance may affect how people allocate further search efforts across options. Specifically, outcome variance and sample size may be associated such that people will allocate more of their search effort to the option with the higher outcome variance, because it represents, ceteris paribus, the riskier option. There is, however, one complication to this hypothesis. In decision from experience studies, people are initially unaware of the options' possible outcomes, and by extension, of the outcome variance. They only gradually learn about the outcomes by engaging in search. Moreover, the relationship between sample size and observed variance is bidirectional. Higher experienced variance may trigger additional search but larger samples also provide the stage for variance to unfold: A rare event is more likely to occur in a large sample than in a small sample. Therefore, the direction of the causal 
relationship between outcome variance and search length could be confounded. A way to deal with this confound is to compare the sample drawn by each participant in each of two classes of options, the one experienced as more risky (variable) and the one experienced as less risky. If experienced outcome variance prompted more search, then people who happen to experience it would search more in this option relative to one in which they did not experience variance. No such asymmetry would emerge when people fail to experience variance in both options.

To summarize: Previous studies of decisions from experience have shown that search effort depends, among other variables, on the cognitive properties of the choosing organism. In contrast, we know relatively little about the possible dependencies of search effort and properties of the choice ecology. Therefore, we make first steps in investigating such dependencies. We focus on two ecological properties-the domain (gain vs. loss) and experienced variance (variance vs. no variance)-and analyze them using two sets of data.

\section{Method}

Our analysis proceeded in two steps. First, we analyzed sample size data from the following published studies: Camilleri and Newell (2010), Hau et al. (2008), Hertwig et al. (2004), Hertwig and Pleskac (2010), and Ungemach, Chater, and Stewart (2009). All data stems from conditions implementing the sampling paradigm: Participants could freely choose how long to sample and how to allocate search across the two payoff distributions. Taking advantage of these data, we can analyze on an aggregate level (averaged across participants) to what extent sample size depends on the choice ecology.

In our analysis' second step, we turned to individual data to examine the degree to which aggregate and individual results converge. Specifically, we took advantage of the data set collected in the Technion Prediction Tournament (TPT; Erev et al., 2010). The TPT is a prediction competition in which different models were fitted to people's choices across 60 problems. The models were then used to predict their choices in 60 new problems. The competition involved two decisions-from-experience paradigms, one of which was the sampling paradigm that we consider here. Each of the 120 fitting and prediction problems represents a choice between a safe option offering a medium payoff $(M)$ with certainty and a risky option offering a high payoff $(\mathrm{H})$ with some probability $(p(\mathrm{H}))$ and a low $(\mathrm{L})$ payoff with the complementary probability. $\mathrm{M}, \mathrm{H}, \mathrm{p}(\mathrm{H})$, and L were generated randomly, and a selection algorithm assured that the 120 problems differed in domain (gains, losses, and mixed payoffs) and probability (high, medium, and low). The resulting set of problems is the largest publicly available data set of the sampling paradigm.

\section{Results}

\subsection{Is there a dependency between domain and sample size?}

We address this question by focusing on choice problems that involve outcomes of the same magnitude (e.g., 32) but different signs (+ vs. -; that is gains vs. losses) within the same study (thus the results are based on the same pool of participants and the same payment regime). We calculated (a) the difference between the aggregate sample sizes for each problem's loss and gain domain, and (b) the relative increase (i.e., the difference divided by the size of the smaller sample). Fig. 1 plots these differences for four problems that were investigated 15 times in independent experiments. Bars that point upward indicate that participants drew larger samples in the loss relative to the gain domain. In 12 of 15 problem pairs, participants

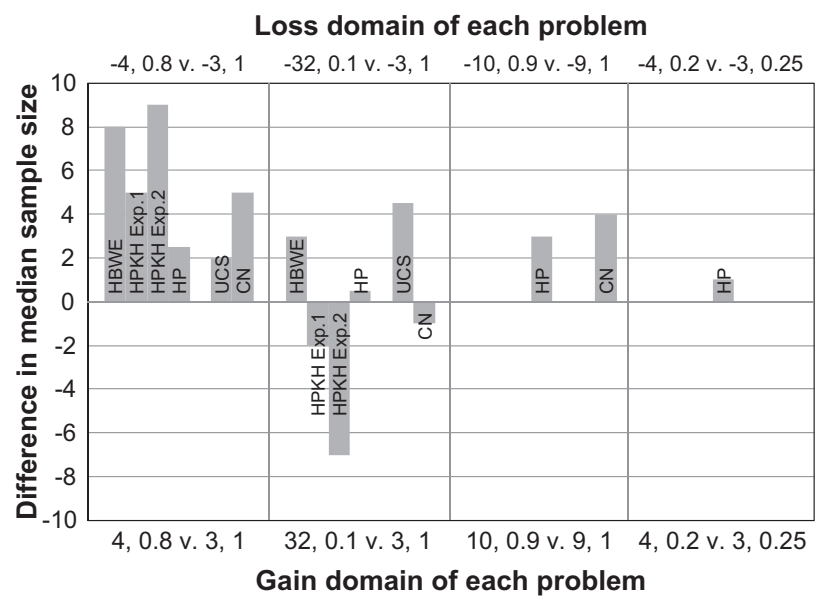

Fig. 1. Difference between median sample size in the loss and gain domain of each problem (Fig. 1). Each bar represents a study and each of the four boxes represents a pair of problems. HBWE: Hertwig et al. (2004); HPKH Exp. 1: Hau et al. (2008) Experiment 1; HPKH Exp. 2: Hau et al. (2008) Experiment 2; HP: Hertwig and Pleskac (2010); UCS: Ungemach et al. (2009); CN: Camilleri and Newell (2010). Problems are described in the header (loss domain) and footer (gain domain) of the figure. For example, the leftmost box shows the difference between sample sizes in the loss and gain domain of a problem that offers a gain or loss of 4 with a .8 probability ( 0 otherwise) or a certain gain or loss of 3 . The leftmost bar shows that Hertwig et al. (2004) found a median sample in the loss domain that was eight observations larger than in the gain domain. 


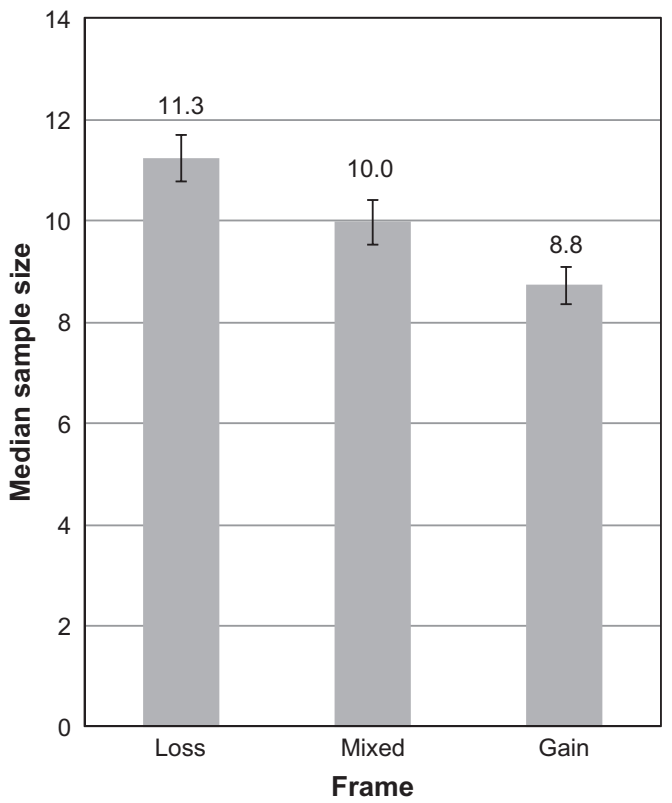

Fig. 2. The median sample sizes in the TPT data set (Erev et al., 2010) as a function of the domain: gain, mixed and loss domain, respectively. Error bars indicate standard errors.

sampled more in the loss domain $(z=2.32, p=.04$, twotailed binomial test). Across studies, samples in the loss domain encompassed, on average, 2.5 ( $\mathrm{SD}=4$ ) observations more than those in the gain domain. In the 12 problems in which participants sampled more in the loss domain, the relative increase in search amounted to, on average, 36\%; across all 15 problems, it amounted to $25 \%$.

Results on the aggregate level indicate that when faced with the prospect of a loss people search more than when experiencing the prospect of a gain. Next, we conducted a similar analysis on the individual level using the TPT data set. The problems therein were not designed to be symmetrical (that is, identical in payoff magnitude but differing in the sign). Yet, on average, the payoff magnitudes in the 40 loss problems and 40 gain problems were nearly identical $\left(\right.$ Mean $_{\text {loss }}=-10.2, \mathrm{SD}_{\text {loss }}=7.7, \quad$ Mean $_{\text {gain }}=10.1$, $\mathrm{SD}_{\text {gain }}=7.1$ ), thus rendering it possible to compare sample sizes across domains. Each participant completed 30 problems: 10 in the gain domain, 10 in the loss domain, and 10 in the mixed domain, respectively. Separately for each participant, we calculated the median sample size in the loss and gain domains, respectively, and compared them using a Wilcoxon Signed Ranks Test. Echoing the results reported in Fig. 1, Fig. 2 shows that people sample more in the loss relative to the gain domain $(M d n=11.25$ vs. 8.75; $z=-3.36, p<0.01, r=-.37)$; the relative increase was 29\%. Fig. 2 also shows the sample size in 40 problems involving mixed outcomes (gains and losses). The median sample size falls in the middle of the two uniform domains, and is significantly larger than the median sample in the gain domain $(z=-1.99, p=.047, r=-.22)$, and smaller than the median sample in the loss domain $(z=-2.33$, $p=0.02, r=-.26$ ).
Finally, a majority (44 of 80 participants, 55\%) sampled, on average, more in the loss than in the gain domain; $30 \%$ (24 of 80 ) showed the reverse regularity, and 15\% (12 of 80) balanced their search effort across domains. We also found that the difference in sample size for those who sample more in the loss than the gain domain was about two times the size of the difference observed among those who preferentially explored the gain domain $\left(\operatorname{diff}_{L-G}=4.3\right.$, $41 \%$, vs. $\left.\operatorname{diff}_{\mathrm{G}-\mathrm{L}}=1.7,16 \%, t(66)=2.6, p<.01\right)$.

\subsubsection{Summary}

Although loss aversion has been used as an explanatory construct across a wide range of findings and phenomena (e.g., endowment effect), its empirical status is somewhat disputed. In fact, in research on description- and experience-based risky choice, its ability to explain actual behavior (choice) in monetary gambles has been questioned (see Erev et al., 2008; Hochman \& Yechiam, 2011). At the same time, Hochman and Yechiam reported evidence that the possibility of losses results in increased autonomic arousal relative to the possibility of gains. Search for information could be understood as an intermediate step between initial arousal and final choice. If so, it is an open question to what extent it will give rise to a loss-gain asymmetry, present in physiological data but disputed in choice data.

In our analyses, we found evidence for this asymmetry on both the aggregate and individual levels. Let us emphasize, however, that nearly half the people either did not allocate their search effort asymmetrically, or showed the opposite asymmetry, that is, searched more in the gain than in the loss domain. The latter's preference in search was much less pronounced in magnitude than the reversed asymmetry. Individual differences in loss aversion have previously been observed both at the behavioral (Frederick, 2005) and neural (Tom et al., 2007) level. Tom et al. concluded that such individual differences in behavioral loss aversion are driven mostly by individual differences in neural sensitivity to potential losses. Future studies may investigate to what extent differences in search effort in the face of losses can be predicted by differences in neural sensitivity to losses. Taken together, the present observations indicate that the domain of choice (loss vs. gain) has a discernible effect on search in decisions from experience.

\subsection{Does experienced variance invoke more search?}

In order to investigate a possible link between experienced variance and search effort, we will focus on the sampling data set of the TPT tournament. Using the total set of 120 problems (40 gain, 40 loss, and 40 mixed gambles), we first examined whether participants experienced variance in the risky option of each choice problem: When a participant experienced more than one kind of outcome from the risky option, we classified this participant as having experienced variance in this problem; otherwise, as having experienced no variance. Then, we calculated the difference between the sample size drawn from the risky and the safe option for each participant (and in each of the 30 problems presented per individual). A positive difference denotes more search in the risky relative to the safe option. 


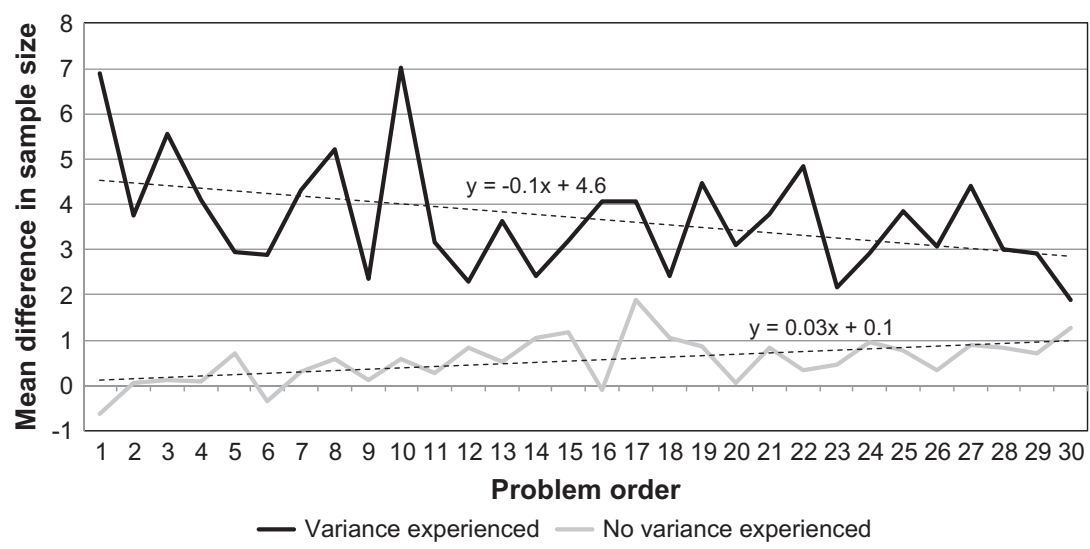

Fig. 3. Mean difference (averaged across participants) in sample size between the risky option and the safe option across 30 problems in the TPT. The graph includes the trend lines for each mean difference and the corresponding linear equation.

Fig. 3 shows that, averaged across all participants, samples were larger in the risky than in the safe option for participants who experienced variance (black curve). In contrast, participants who failed to experience the risky option's variance allocated nearly equal search effort to both options (gray curve). Participants who experienced variance sampled 3.7 observations more from the risky than the safe option, relative to an increase of 0.5 observations for participants who experienced no variance. At the individual level, 52 of 75 (69\%) participants sampled more from the risky than the safe option when they experienced variance relative to when they did not (Wilcoxon Signed Ranks Test, $z=-3.58, p<0.01, r=-.41)$.

Fig. 3 also shows that participants who did not experience variance drew slightly more observations from the risky relative to the safe option (otherwise the trend line would be identical with the $x$-axis), even though both options appeared equally invariant. Moreover, this pattern appears more pronounced in later relative to earlier problems. At the same time, the search gap (between the risky and safe option, respectively) among those participants who experienced variance appears to decrease across trials. One interpretation of these trends is that participants may have learnt the structure of the choice problems over time (a finding also reported by Hadar \& Fox, 2009; Study 2 ). That is, they recognized that all problems consist of a risky and a safe option, and that the risky option includes a high and low payoff, whereas the safe options come with a medium payoff. Across trials, people may learn to eyeball what a medium payoff (indicative of the safe option) is and what a low and high payoff (indicative of a risky option) is. Being thus cognizant of the structure of the problem may mean that over time those who did not encounter variance in a given problem may still search somewhat more in what they suspect to be the risky option, spurred by the expectation that it may ultimately involve another distinct outcome. Following a similar logic, those who encountered variance in a given problem, in contrast, may sample increasingly less from the risky option because on the first encounter of the second outcome they are fully aware of the range of outcomes and can even guestimate their probabilities; no further search is necessary. ${ }^{2}$ This interpretation-decision makers learn the structure of the problems and with learning seek (require) less information-is consistent with Fig. 4. It depicts the average total sample size (safe and risky option) across the sequence of 30 choice problems. On average, mean sample size gradually drops from 25.5 samples (1st problem) to 9.1 samples (30th problem). Out of 80 participants, 54 (67.5\%) people display such a gradually decreasing trend, 16 (20\%) and 10 (12.5\%) show increasing and constant trends, respectively.

\subsubsection{Summary}

People sample more or less as a function of their experience of variance or lack thereof. Specifically, the experience of variance results in larger search gaps between risky and safe options relative to problems in which participants failed to experience variance in the risky option (Fig. 3). In the latter, search was nearly equally allocated between safe and risky options. What is the cause and what is the effect in this pattern? Does the experienced variance trigger more search? Or, alternatively, are ardent explorers doomed to experience variance? It is difficult to discern between these two possibilities. We believe that the first is more plausible for the following reason: Most people explore a lot in one problem and little in the next, and variability in search effort is considerable (Fig. 5). This suggests that search is more shaped by the experience (e.g. of variance) in the current problem and less so by a preset and gamble-independent aspiration level concerning length of search.

\section{How does search affect choice?}

Search, like choice, is a distinct behavioral and cognitive process in its own right. It provides us with a window on

\footnotetext{
${ }^{2}$ The probability of the outcome they encounter once can be inferred to be the complement of the estimated probability of the outcome encountered several times.
} 


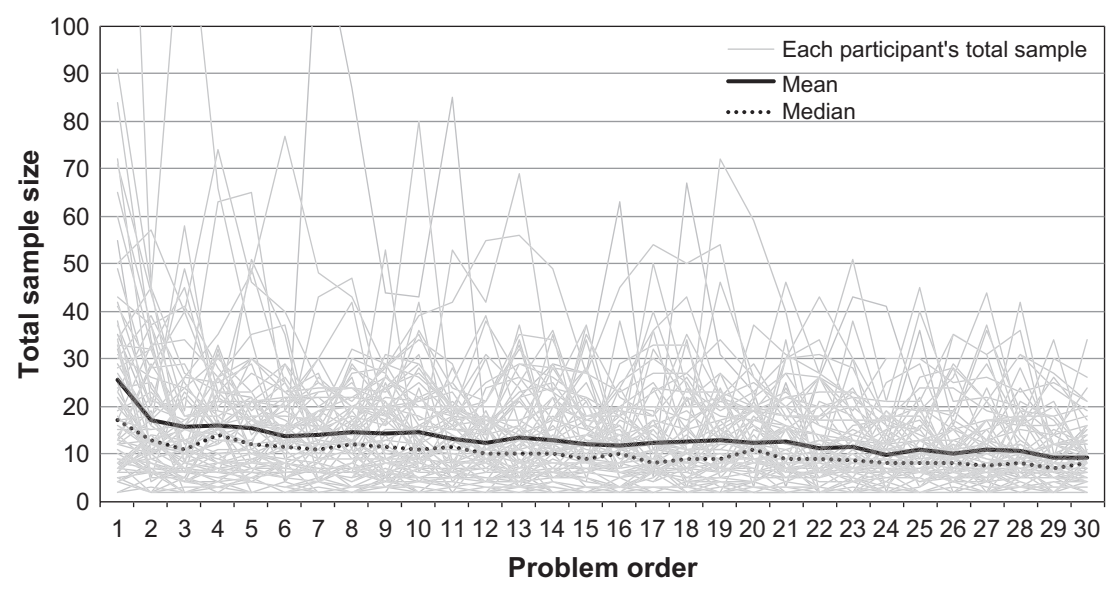

Fig. 4. Combined sample size in the risky and safe option by problem order, separately for each participant. The $y$-axis is truncated at 100 excluding three outliers. The solid black and the dashed black lines represent the mean and median sample sizes, respectively, across 80 participants.

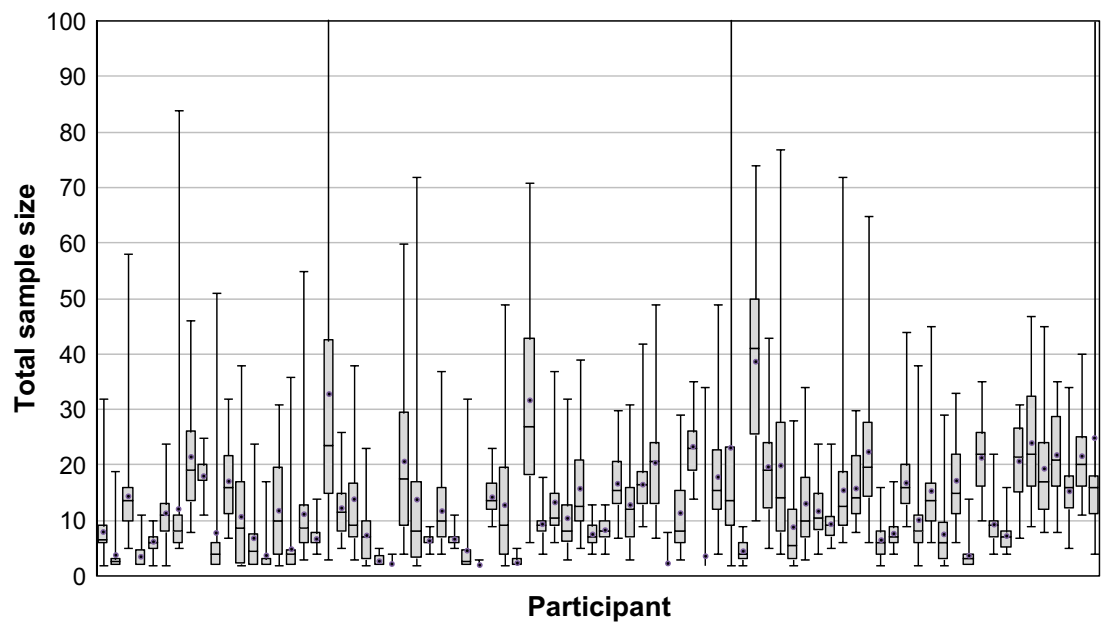

Fig. 5. Variance in total sample size (risky and safe option). Each box represents the variance for one of the 80 participants. The size of the box denotes the 2nd and 3rd quartiles separated by the median. Dots denote the mean sample size and whiskers denote maximum and minimum values. The $y$-axis is truncated at 100 excluding three outlying maximum values.

the mind, and on people's fears and hopes. Yet, one question naturally arises: Do the observed effects in search translate into measurable effects in choice? One way to answer this question is by analyzing the systematic difference between description- and experienced-based choices. The size of the description-experience gap (Hertwig \& Erev, 2009) is markedly (but not exclusively) determined by sample size. For instance, by greatly increasing the financial stakes, Hau et al. (2008) doubled participants' search efforts (Experiment 2: median of 33 samples relative to Hertwig et al., 2004, median of 15 samples) and found that the description-experience gap was reduced but not eliminated. Relative to such large differences in search effort, the obtained differences between gain vs. loss domain and safe vs. risky option are small. Yet, can they possibly affect choice? In other words, could it be that the description-experience gap is, for instance, larger for choices in the gain domain relative to the loss domain because participants sample more from the latter domain?
Using the TPT data set, we determined the descriptionexperience gap for each problem in the gain and loss domains by calculating the average (absolute) difference between the proportion of choices of the risky options in the description (i.e., choices by other participants in the TPT) and the experience conditions. We included only those participants who exhibited a gain-loss asymmetry in search, irrespective of whether or not they experienced variance. The gaps proved to be essentially identical in size (gain vs. loss domain: $34 \%$ vs. $32 \%$ gap; $t(78)=-.47$, $p=.64 .^{3}$

\footnotetext{
${ }^{3}$ Yechiam and Telpaz (2011) suggested that choices in the loss domain are more in line with expected-value maximization than choices in the gain domain, a possible consequence of heightened vigilance when encountering and evaluating losses. We analyzed, for each participant the proportion of EV maximization choices (assuming the experienced sample information) and found no difference between the loss (83\%) and gain (84\%) domains, $t(79)=-.38, p=.70$.
} 
We conducted a similar analysis for variance, and determined the proportion of risky choices, separately for those participants who did and did not experience variance. Note, however, that participants who happen not to experience outcome variance choose between two invariant amounts. Therefore, their choices will depend on the structure of the problem, particularly on whether the problem involves rare events (i.e., in 80 of the 120 problems), and the analysis needs to take this into account. Specifically, the problems in the TPT involve a choice between a safe option offering a medium amount and a risky option offering a high amount with probability $p$ (high) and a low amount otherwise. When $p$ (high) $<0.1$, participants who did not experience variance are likely to choose between a medium amount (safe option) and a low amount (risky option). On average, the proportion of risky choices in this class of problems was $10 \%$ (no variance experienced) and 73\% (variance experienced; $t(32)=-13.35, p<.001$ ), respectively. Conversely, when $p$ (high) $>0.9$, participants who encountered no variance choose between a medium (safe option) and a high amount (risky option). In this class of problems, the proportion of risky choices was 78\% (no variance experienced) and 20\% (variance experienced; $t(40)=17.44, p<.001)$. Unlike in the domain of choice, search effort has substantial implications for choice involving rare events: lack of experienced variance can lead to a strong preference for the risky or the safe option, depending on whether the common outcome in the risky option represents an attractive or an unattractive result.

\section{Conclusions}

In decisions from experience, people are the masters of their information search insofar as they control how they distribute their attention over options and for how long they do so. Previously, research has focused on the psychological endowment of the information foragers in experience-based decision making, for instance, in terms of short-term memory capacity (Rakow et al., 2008), and the ability to think rationally (Lejarraga, 2010). Less progress had been made in understanding how ecological properties shape search. In our analysis, we focused on two properties: gain vs. loss domain and variance. We found that both properties have some moderate impact on how long people search. Our results suggest that loss aversion and risk aversion may not be limited to choice but also leave their traces in search.

One interpretation of our findings is that search effort reflects people's vigilance toward environmental stimuli. When facing the prospect of losses most people appear to invest more effort exploring potentially disadvantageous than advantageous payoff distributions. Heightened vigilance in the face of potential losses is a notion that has been proposed by Yechiam and Telpaz (2011). Their attentional account conflicts with Wakker (2010) who suggested that losses reduce the reliability of a person's choices. In relation to these two views, our results suggest that losses systematically boost search; in addition, our findings of similar description-experience gaps in the gain and loss domain is not suggestive of less reliable choices in the loss domain (but see also Footnote 3).

Similarly to the prospect of losses, when facing risk (experience of variation), people appear to allocate more search effort toward those riskier options, relative to safer options. Although not large, the effects are noticeable. Moreover, they are consistent with observations such as the finding that individuals spend more time deciding among losses than among gains (Gonzalez et al., 2005; Payne, Bettman, \& Johnson, 1993), and explore options longer when they bargain for losses than when they bargain for gains (Camerer, Johnson, Rymon, \& Sen, 1993).

In Cognition in the Wild, Hutchins (1995) wrote "any cognitive psychologist would be happy enough to be able to look directly at the content of the cognitive system" (p. 129). He also proposed that with the study of "systems of socially distributed cognition we can step inside the cognitive system." We agree. But next to studying socially distributed cognition, we can also take advantage of search for information in external worlds to thus directly observe the operation of the cognitive system. Although some processes inside people's heads still remain obscured-for instance, how they integrate the obtained information into a judgment-the traces of loss and risk aversion appear to be discernable in external search.

\section{Acknowledgments}

We thank Laura Wiles for editing the manuscript, the Swiss National Science Foundation for a grant to the second author (100014-126558); and the National Institute for Occupational Safety and Health (Centers for Disease Control and Prevention, Award number: 200-200931403) for support to the first and third authors.

\section{References}

Benartzi, S., \& Thaler, R. H. (1995). Myopic loss-aversion and the equity premium puzzle. Quarterly Journal of Economics, 110, 73-92.

Busemeyer, J. R., \& Townsend, J. T. (1993). Decision field theory: A dynamic-cognitive approach to decision making in an uncertain environment. Psychological Review, 100, 432-459.

Camerer, C. I., Babcock, L., Loewenstein, G., \& Thaler, R. H. (1997). Labor supply of New York City cabdrivers: One day at a time. Quarterly Journal of Economics, 112, 407-441. http://dx.doi.org/10.1162/ 003355397555244.

Camerer, C. F., Johnson, E. J., Rymon, T., \& Sen, S. (1993). Cognition and framing in sequential bargaining for gains and losses. In K. Binmore, A. Kirman, \& P. Tani (Eds.), Frontiers of game theory (pp. 27-47). Cambridge MA: MIT Press.

Camilleri, A. R., \& Newell, B. R. (2010). Description- and experience-based choice: Does equivalent information equal equivalent choice? Acta Psychologica, 136, 276-284. http://dx.doi.org/10.1016/ j.actpsy.2010.11.00.

Edwards, W. (1961). Probability learning in 1000 trials. Journal of Experimental Psychology, 62, 385-394. http://dx.doi.org/10.1037/ h0041970.

Erev, I., \& Barron, G. (2005). On adaptation, maximization and reinforcement learning among cognitive strategies. Psychological Review, 112, 912-931.

Erev, I., Ert, E., Roth, A. E., Haruvy, E., Herzog, S. M., Hau, R., et al. (2010). A choice prediction competition: Choices from experience and from description. Journal of Behavioral Decision Making, 23, 15-47. http:// dx.doi.org/10.1002/bdm.683.

Erev, I., Ert, E., \& Yechiam, E. (2008). Loss aversion, diminishing sensitivity, and the effect of experience on repeated decisions. Journal of Behavioral Decision Making, 21, 575-597. http://dx.doi.org/10.1002/ bdm.602. 
Frederick, S. (2005). Cognitive reflection and decision making. Journal of Economic Perspectives, 19, 25-42. http://dx.doi.org/10.1257/ 089533005775196732

Gigerenzer, G., Hertwig, R., van den Broek, E., Fasolo, B., \& Katsikopoulos, K. V. (2005). "A 30\% chance of rain tomorrow": How does the public understand probabilistic weather forecasts? Risk Analysis, 25, 623-629. http://dx.doi.org/10.1111/j.1539-6924.2005.00608.x.

Gonzalez, C., Dana, J., Koshino, H., \& Just, M. (2005). The framing effect and risky decisions: Examining cognitive functions with fMRI. Journal of Economic Psychology, 26, 1-20. http://dx.doi.org/10.1016/j.joep. 2004.08.004

Hadar, L., \& Fox, C. (2009). Information asymmetry in decision from description versus decision from experience. Judgment and Decision Making, 4, 317-325.

Hau, R., Pleskac, T. J., \& Hertwig, R. (2010). Decisions from experience and statistical probabilities: Why they trigger different choices than a priori probabilities. Journal of Behavioral Decision Making, 23, 48-68. http://dx.doi.org/10.1002/bdm.665.

Hau, R., Pleskac, T. J., Kiefer, J., \& Hertwig, R. (2008). The descriptionexperience gap in risky choice: The role of sample size and experienced probabilities. Journal of Behavioral Decision Making, 21, 493-518. http://dx.doi.org/10.1002/bdm.598.

Hertwig, R., Barron, G., Weber, E. U., \& Erev, I. (2004). Decisions from experience and the effect of rare events in risky choice. Psychological Science, 15, 534-539. http://dx.doi.org/10.1111/j.09567976.2004.00715.x.

Hertwig, R., \& Erev, I. (2009). The description-experience gap in risky choice. Trends in Cognitive Sciences, 13, 517-523. http://dx.doi.org/ 10.1016/j.tics.2009.09.004.

Hertwig, R., \& Pleskac, T. J. (2010). Decisions from experience: Why small samples? Cognition, 115, 225-237. http://dx.doi.org/10.1016/ j.cognition.2009.12.009.

Hertwig, R., \& Pleskac, T. J. (2008). The game of life: How small samples render choice simpler. In N. Chater \& M. Oaksford (Eds.), The probabilistic mind: Prospects for Bayesian cognitive science (pp. 209-236). Oxford, UK: Oxford University Press.

Hills, T. T., \& Hertwig, R. (2010). Information search and decisions from experience: Does our patterns of sampling foreshadow our decisions? Psychological Science, 21, 1787-1792. http://dx.doi.org/10.1177/ 0956797610387443.

Hochman, G., \& Yechiam, E. (2011). Loss aversion in the eye and in the heart: The autonomic nervous system's responses to losses. Journal of Behavioral Decision Making, 24, 140-156. http://dx.doi.org/10.1002/ bdm.692.

Hoffrage, U., Weber, A., Hertwig, R., \& Chase, V. M. (2003). How to keep children safe in traffic: Find the daredevils early. Journal of Experimental Psychology: Applied, 9, 249-260. http://dx.doi.org/ 10.1037/1076-898X.9.4.249.

Hutchins, E. (1995). Cognition in the wild. MIT Press.

Kahneman, D., Knetsch, J. L., \& Thaler, R. H. (1990). Experimental tests of the endowment effect and the Coase theorem. Journal of Political Economy, 98, 1325-1348.

Kahneman, D., \& Tversky, A. (1979). Prospect theory: An analysis of decision under risk. Econometrica, 47, 263-292. http://dx.doi.org/ $10.2307 / 1914185$.

Knight, F. H. (1921). Risk, uncertainty, and profit. Boston, MA: Houghton Mifflin.

Lejarraga, T. (2010). When experience is better than description: Time delays and complexity. Journal of Behavioral Decision Making, 23, 100-116. http://dx.doi.org/10.1002/bdm.666.
Ludvig, E. A., \& Spetch, M. L. (2011). Of black swans and tossed coins: Is the description-experience gap in risky choice limited to rare events? PLOS ONE, 6, 6. http://dx.doi.org/10.1371/journal.pone.0020262.

Markowitz, H. (1952). Portfolio selection. Journal of Finance, 7, 77-91.

Myers, J. L., \& Sadler, E. (1960). Effects of range of payoffs as a variable in risk taking. Journal of Experimental Psychology, 60, 306-309.

Newell, A., \& Simon, H. A. (1972). Human problem solving. Englewood Cliffs, NJ: Prentice Hall.

Payne, J. W., Bettman, J. R., \& Johnson, E. J. (1993). The adaptive decision maker. Cambridge, England: Cambridge University Press.

Rakow, T., Demes, K. A., \& Newell, B. R. (2008). Biased samples not mode of presentation: Re-examining the apparent underweighting of rare events in experience-based choice. Organizational Behavior and Human Decision Processes, 106, 168-179. http://dx.doi.org/10.1016/ j.obhdp. 2008.02.001.

Samuelson, W., \& Zeckhauser, R. (1988). Status quo bias in decision making. Journal of Risk and Uncertainty, 1, 7-59.

Sharpe, W. F. (1964). Capital asset prices: A theory of market equilibrium under conditions of risk. Journal of Finance, 19, 425-442.

Simon, H. A. (1956). Rational choice and the structure of the environment. Psychological Review, 63, 129-138.

Simon, H. A. (1990). Invariants of human behavior. Annual Review of Psychology, 41, 1-20. http://dx.doi.org/10.1146/annurev.ps. 41.020190.000245.

Simon, H. A. (1996). Models of my life. Cambridge, MA: MIT Press.

Thaler, R. (1980). Toward a positive theory of consumer choice. Journal of Economic Behavior and Organization, 1, 39-60.

Todd, P. M., Hills, T. T., \& Robbins, T. W. (Eds.) (2012). Cognitive search: Evolution, algorithms, and the brain. Strüngmann Forum Reports (Vol. 9). Cambridge, MA: MIT Press.

Todd, P. M., Penke, L., Fasolo, B., \& Lenton, A. P. (2007). Different cognitive processes underlie human mate choices and mate preferences. Proceedings of the National Academy of Sciences, 104, 15011-15016.

Tom, S. M., Fox, C. R., Trepel, C., \& Poldrack, R. A. (2007). The neural basis of loss aversion in decision making under risk. Science, 315, 515-518. http://dx.doi.org/10.1126/science.1134239.

Tversky, A., \& Kahneman, D. (1992). Advances in prospect theory: Cumulative representation of uncertainty. Journal of Risk and Uncertainty, 5, 297-323.

Ungemach, C., Chater, N., \& Stewart, N. (2009). Are probabilities overweighted or underweighted when rare outcomes are experienced (rarely)? Psychological Science, 20, 473-479. http:// dx.doi.org/10.1111/j.1467-9280.2009.02319.x.

Von Neumann, J., \& Morgenstern, O. (1947). Theory of games and economic behavior (2nd ed.). Princeton, NJ: Princeton University Press.

Wakker, P. P. (2010). Prospect theory: For risk and ambiguity. Cambridge University Press.

Weber, E. U., Shafir, S., \& Blais, A. (2004). Predicting risk-sensitivity in humans and lower animals: Risk as variance or coefficient of variation. Psychological Review, 111, 430-445. http://dx.doi.org/ 10.1037/0033-295X111.2.430.

Willemsen, M. C., \& Johnson, E. J. (2010). Visiting the decision factory: Observing cognition with MouselabWEB and other information acquisition methods. In M. Schulte-Mecklenbeck, A. Kühberger, \& R. Ranyard (Eds.), A handbook of process tracing methods for decision research (pp. 21-42). New York: Taylor \& Francis.

Yechiam, E., \& Telpaz, A. (2011). Losses induce consistency in risk taking even without loss aversion. Journal of Behavioral Decision Making. http://dx.doi.org/10.1002/bdm.758. 